\title{
CASE REPORT \\ Subacute delayed ascending myelopathy: not just a post-traumatic disorder
}

\author{
AC $\operatorname{Tan}^{1}, \mathrm{~K} \mathrm{Ng}^{2,3}, \mathrm{~A} \mathrm{Newey}^{3,4}$ and G Leong ${ }^{1,3}$
}

Study design: Case report.

Objectives: Subacute delayed ascending myelopathy (SDAM), also known as subacute post-traumatic ascending myelopathy, is a rare early neurological complication of spinal cord injury (SCI), and the aetiology, pathogenesis and optimal management of this condition are poorly understood. The radiological features together with the clinical picture appear to be the most useful. We aim to increase awareness and further characterise SDAM.

Setting: Spinal Cord Injuries Unit, Royal North Shore Hospital, Sydney, Australia.

Methods and Results: We report two cases with radiological findings consistent with SDAM, and review the literature. Only a small number of cases have been reported and importantly, we report the first case occurring following a non-traumatic SCl. There are several hypotheses regarding pathogenesis, with several factors in our cases implicating a vascular mechanism.

Conclusion: There is a lack of data characterising SDAM, and ascending myelopathy in a stable SCI should alert the clinician. Importantly, we propose that SDAM is the appropriate terminology.

Spinal Cord (2014) 52, S11-S13; doi:10.1038/sc.2014.82

\section{INTRODUCTION}

Subacute delayed ascending myelopathy (SDAM) is a rare, underrecognised neurological complication of spinal cord injury (SCI), defined as a delayed, subacute loss of spinal cord and nerve root function greater than four spinal segments above the original lesion unrelated to mechanical instability ${ }^{1}$ (Supplementary Table).

\section{CASE REPORT}

\section{Case 1}

A 16-year-old male sustained a T12 American Spinal Injuries Association Impairment Scale (AIS) A spinal injury in a motocross accident. Imaging revealed an L1 burst fracture with a large retropulsed fragment causing significant compression of the conus medullaris and haemorrhage within the cord at the T12/L1 level. Early posterior spinal decompression and fixation was performed. A thoraco-lumbar-sacral orthosis was worn for six weeks. His postoperative period was complicated on day 2 by a pulmonary embolism and bronchopneumonia. He was mobilised on day 10 into a manual wheelchair.

On day 11, the patient reported thoracic numbness and paradoxical breathing was noted, with reduced light touch sensation to T4 bilaterally. A low-grade pyrexia to $37.8^{\circ} \mathrm{C}$ was noted in the two days prior. Magnetic resonance imaging (MRI) revealed high signal from the conus to the cervico-thoracic junction, centrally within the cord (Figures 1 and 2). Inflammatory markers were negative. High-dose corticosteroid ( $1 \mathrm{~g}$ intravenous methylprednisolone daily) was administered for 8 days. Repeat MRI 1 week later showed persistent but less marked high signal within the thoracic cord. Two weeks later, light touch sensation had improved to T6 (right) and T7 (left). Pinprick sensation was at T4 (right) and T7 (left). The SCI remained stable at T3 AIS A.

\section{Case 2}

A 65-year-old male underwent an Ivor-Lewis oesophagectomy for oesophageal adenocarcinoma. Post-operatively he had no motor or sensory function of his lower limbs (T3 AIS A). MRI revealed a central spinal cord infarct at T9/T10. Steroids (4 mg intravenous dexamethasone four times daily) were given for 3 days. His course was complicated by bilateral below-knee deep vein thromboses, treated with anticoagulation.

Six weeks after the initial injury, the patient developed right arm numbness. Examination revealed increased motor and sensory deficit, at C7 AIS A. MRI revealed new patchy hyperintensity on T1-weighted imaging from $\mathrm{T} 6$ to the conus and new T2-hyperintensity from C4-T12 (Figure 3). Haemorrhagic infarction was contemplated, with anticoagulation ceased and an inferior vena cava filter inserted. Repeat MRI 8 days later showed no significant change, and steroids ( $1 \mathrm{~g}$ intravenous methylprednisolone daily) were administered for 5 days. There was no improvement in the patient's SCI.

\section{DISCUSSION}

\section{Clinical features}

SDAM has been previously described as subacute post-traumatic ascending myelopathy and subacute progressive ascending myelopathy. To our knowledge, we report the first case following a nontraumatic SCI and that SDAM is the most appropriate terminology.

${ }^{1}$ Spinal Cord Injuries Unit, Royal North Shore Hospital, Sydney, NSW, Australia; ${ }^{2}$ Department of Neurology, Royal North Shore Hospital, Sydney, NSW, Australia; ${ }^{3}$ Sydney Medical School, The University of Sydney, Sydney, NSW, Australia and ${ }^{4}$ Department of Radiology, Royal North Shore Hospital, Sydney, NSW, Australia

Correspondence: Dr G Leong, Spinal Cord Injuries Unit, Royal North Shore Hospital, Sydney, NSW 2065, Australia.

E-mail: grace.leong@health.nsw.gov.au

Received 26 February 2014; revised 10 April 2014; accepted 28 April 2014 
a

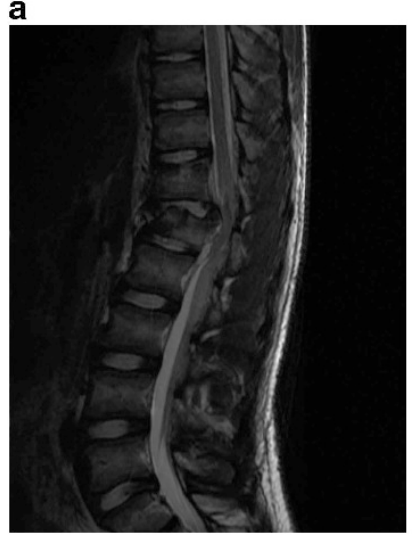

b

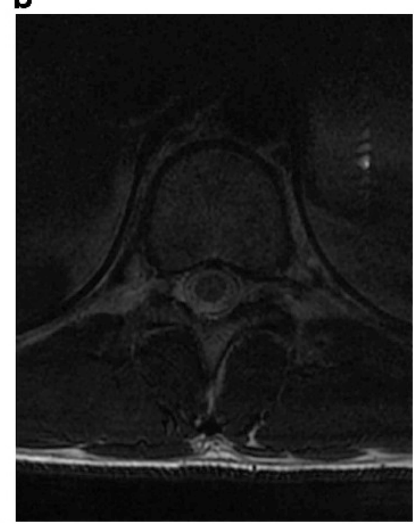

Figure 1 Patient 1 -sagittal T2-weighted image (a) through the thoracolumbar junction performed on day 1 , hours after the injury showed the fracture disclocation at T12/L1 and compressed conus with high signal extending one vertebral body above the compressed cord. An axial T2weighted image (b) two vertebral bodies above the compressed conus shows a normal appearance of the cord.
There are several unsubstantiated hypotheses regarding pathogenesis, including anterior spinal artery thrombosis or ischaemia, venous thrombosis, fibrocartilaginous embolism, an autoimmune or inflammatory reaction, altered CSF drainage or uncontrolled secondary injury. ${ }^{1}$ Several factors in our cases implicate a vascular mechanism, as with reports by Aito et al., ${ }^{2}$ and Schmidt. ${ }^{3}$ Case 1 had an injury at the thoraco-lumbar junction, where the artery of Adamkiewicz frequently enters, a potential area of intra-operative interference and ischaemia. The prior pulmonary embolism, and relative hypoxia or increased vena caval pressure may have impaired cord venous drainage. Any factor exacerbating spinal venous hypertension and/or arterial hypotension, may lead to reduced cord perfusion and SDAM. ${ }^{3}$ However, cord ischaemia may be chronic rather than large vessel in aetiology because of the progressive deterioration and response to a variety of interventions.

\section{Radiological features}

SDAM is characterised by T2-hyperintensity in the central spinal cord, often with mild cord expansion. On T1-weighted imaging, changes are usually minimal, unless complicated by haemorrhage resulting in heterogenous T1-hyperintensity (as in Case 2). Following gadolinium a

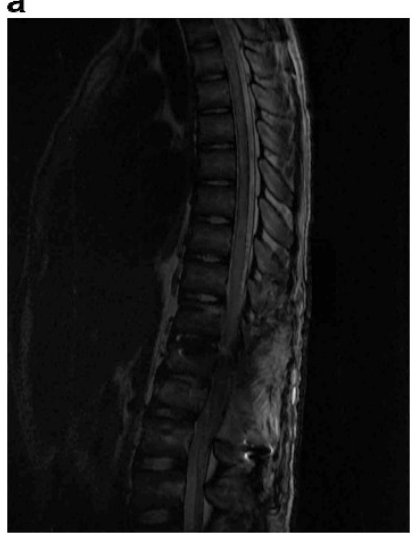

b

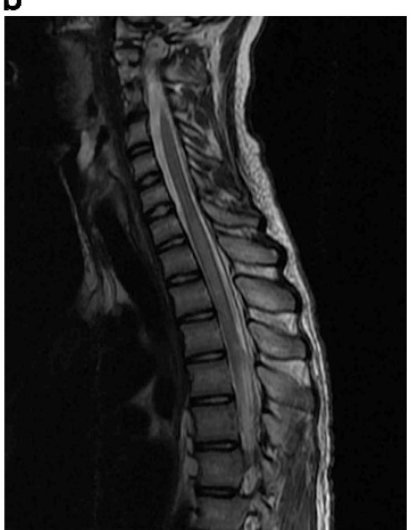

C

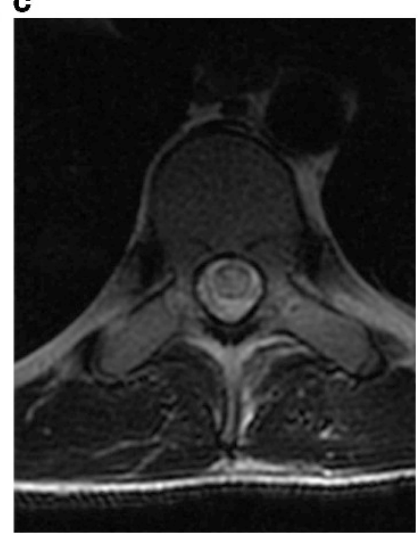

Figure 2 Patient 1 - on day 11 when new neurological symptoms developed, a repeat MRI of the spine was performed. Sagittal T2-weighted image (a) of the thoracolumbar junction and (b) cervico-thoracic junction demonstrated new high signal extending from the conus to the cervico-thoracic junction. Axial T2-weighted image (c) demonstrated the T2 high signal expanding the central thoracic cord with a thin rim of T2 hypointensity.

a

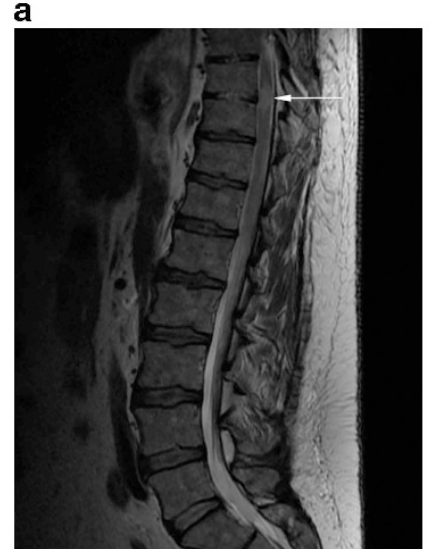

b

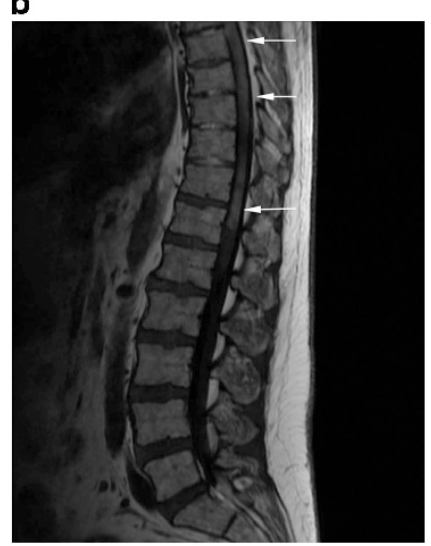

c

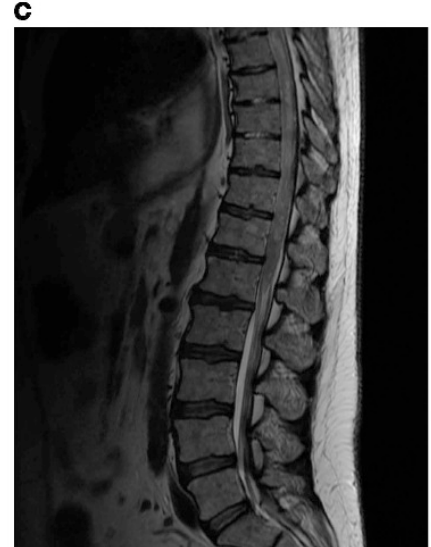

d

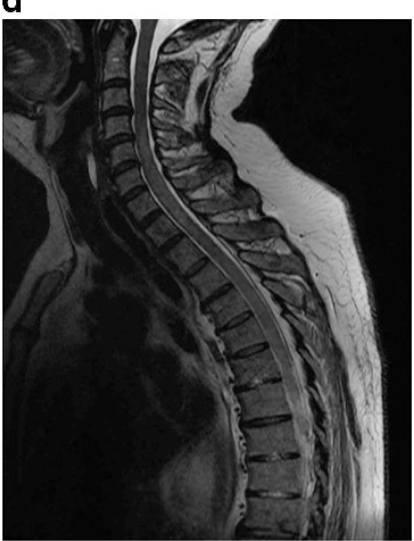

Figure 3 Patient 2-sagittal T2-weighted image (a) of the thoracolumbar junction showed focal central T2-hyperintensity in the central cord at T9/10 thought to represent ischaemia. Follow up MRI was performed after neurological deterioration 6 weeks later. Sagittal T1-weighted image (b) demonstrated patchy T1 high signal in the thoracic cord, compatible with haemorrhage. Sagittal T2-weighted image (c and d) shows diffuse oedema in the thoracic cord. 
there can be patchy intramedullary enhancement (both cases). Prominent T2 flow voids on the dorsal surface of the cord (as in Case 1) and prominence of the epidural venous plexus may implicate venous hypertensive myelopathy, due to thrombosis or obstruction. Radiological confirmation with MRI is an important criterion for the diagnosis of SDAM. ${ }^{4}$ The role of other imaging modalities, such as FDG-PET, in the assessment of SDAM remains unclear.

\section{Prognosis}

A majority of the documented cases of SDAM involve improvement in final neurological function, although not to their baseline. There have been various treatment trials despite the lack of guidelines, with methyprednisolone often administered empirically. There is no consensus on dose or length of treatment ${ }^{3}$ or correlation with response. Cord untethering, duroplasty and cordectomy have also been reported to improve outcome. ${ }^{5}$

\section{CONCLUSION}

An ascending myelopathy after a stable mechanical SCI should alert the clinician. Presently, there are no accepted criteria for diagnosis, but a supportive clinical picture with radiological confirmation and exclusion of other causes appears most important.

\section{CONFLICT OF INTEREST}

The authors declare no conflict of interest.

1 Belanger E, Picard C, Lacerte D, Lavallee P, Levi ADO. Subacute posttraumatic ascending myelopathy after spinal cord injury. J Neurosurg 2000; 93: 294-299.

2 Aito S, El Masry WS, Gerner HJ, Di Lorenzo N, Pellicano G, D’Andrea M et al. Ascending myelopathy in the early stage of spinal cord injury. Spinal Cord 1999; 37: 617-623.

3 Schmidt BJ. Subacute delayed ascending myelopathy after low spine injury: case report and evidence of a vascular mechanism. Spinal Cord 2006; 44: 322-325.

4 Planner AC, Pretorius PM, Graham A, Meagher TM. Subacute progressive ascending myelopathy following spinal cord injury: MRI appearances and clinical presentation. Spinal Cord 2008; 46: 140-144.

5 Meagher TM, Belci M, Lopez de Heredia L, Ansorge O, Jamous A, Saif M et al. Resolution of SPAM following cordectomy: implications for understanding pathophysiology. Spinal Cord 2012; 50: 638-640.

Supplementary Information accompanies this paper on the Spinal Cord website (http://www.nature.com/sc) 